\title{
Critical appraisal of pemetrexed in the treatment of NSCLC and metastatic pulmonary nodules
}

This article was published in the following Dove Press journal:

OncoTargets and Therapy

6 June 2014

Number of times this article has been viewed

Xin Li

Sen Wei

Jun Chen

Tianjin Key Laboratory of Lung Cancer Metastasis and

Tumor Microenvironment, Tianjin Lung Cancer Institute, Department of Lung Cancer Surgery, Tianjin Medical University General Hospital, Heping District, Tianjin,

People's Republic of China
Correspondence: Jun Chen No 154,Anshan Road, Heping District, Tianjin, People's Republic of China 300052 Email huntercj2004@yahoo.com

\begin{abstract}
Pemetrexed, a new multitarget antifolate antineoplastic agent, has significantly improved the overall survival in nonsquamous non-small-cell lung cancer patients. Presently, pemetrexed is recommended for first line treatment in combination with platinum derivatives, for second line treatment as a single agent and, more recently, as maintenance treatment after first line chemotherapy. In this article we critically appraise the status of pemetrexed including pharmacodynamics, pharmacokinetics, toxicity, and the cost effectiveness of pemetrexed, as well as the predictive biomarkers for pemetrexed based chemotherapy.
\end{abstract}

Keywords: chemotherapy, non-small-cell lung cancer, pemetrexed

\section{Introduction}

Lung cancer has become one of the leading causes of cancer-related mortality in developed countries. Non-small-cell lung cancer (NSCLC), which includes squamous cell carcinoma, adenocarcinoma, and large cell carcinoma, is the most common form of lung cancer, accounting for approximately $80 \%$ of all lung cancers. ${ }^{1,2}$ Unfortunately, most NSCLC patients are diagnosed with advanced disease which cannot be successfully treated by surgery. In addition, significant proportions of patients diagnosed with early stage disease eventually relapse and metastasize. Chemotherapy has played a central role in the treatment of patients with NSCLC for over 30 years. In 1995, a meta-analysis demonstrated that cisplatin based doublet chemotherapy, for a maximum of six cycles in the absence of unacceptable toxicity or progressive disease, produced a significant survival benefit in patients with advanced NSCLC. ${ }^{3}$ In the 1990s, various chemotherapeutic agents, including docetaxel, paclitaxel, vinorelbine, and gemcitabine, were developed and became available for the treatment of NSCLC. However, in NSCLC response rates to these treatments were often lower than in other cancers and treatment did not increase long-term survival, particularly for late stage NSCLC. For example, platinum doublet therapy reached a therapeutic plateau with an objective response rate of $30 \%-40 \%$ and a median survival time (MST) of $8-10$ months for patients with stage IIIB or IV disease. ${ }^{4}$ Treatment outcomes for NSCLC patients are still considered unsatisfactory. To improve this situation, various new antineoplastic agents have been proposed for the treatment of this disease. Pemetrexed, a relatively new antifolate antineoplastic agent, has improved the overall survival of nonsquamous NSCLC patients. Presently, pemetrexed is accepted for first line treatment in combination with platinum derivatives, for second line treatment as a single agent and, more recently, as maintenance treatment after first line chemotherapy. In this article we critically appraise the status of pemetrexed. ${ }^{5}$ 


\section{Recommendations for pemetrexed}

Pemetrexed is a multitargeted antifolate agent, developed by Eli Lilly and Company (Indianapolis, IN, USA) and registered for the treatment of malignant pleural mesothelioma and advanced nonsquamous NSCLC. ${ }^{6}$ Currently, pemetrexed is employed in combination with platinum derivatives for first line induction treatment and as single agent for second and subsequent lines of chemotherapy; moreover, it can be administered as maintenance treatment after first line chemotherapy. Due to its effectiveness and its mild toxicity, pemetrexed is widely employed. Additionally, its specific action against the nonsquamous histotype makes it a useful example of a histology-specific approach in oncology.

According to the American Society of Clinical Oncology Clinical Practice Guidelines on Chemotherapy for stage IV NSCLC: ${ }^{-7-9}$ Recommendations were based on the treatment strategies that improve overall survival (OS). Treatments that improve only progression-free survival (PFS) prompted scrutiny of toxicity and quality of life. In NSCLC patients with stage IV, first-line cytotoxic chemotherapy should be stopped at disease progression or after 4 cycles, in patients whose disease is stable but not responding to treatment. Two-drug cytotoxic combinations should be administered for not more than six cycles. For those with stable disease or response after four cycles, immediate treatment with an alternative, single-agent chemotherapy may be considered, such as pemetrexed in patients with non-squamous histology, docetaxel in unselected patients or erlotinib in selected patients. Limitations of these data are such that a break from cytotoxic chemotherapy after a fixed course is also acceptable, with initiation of second-line chemotherapy at disease progression. Erlotinib and gefitinib are recommended for first line treatment of patients with stage IV epidermal growth factor receptor mutated tumors. Docetaxel, erlotinib, gefitinib (except in Europe), or pemetrexed are recommended as second line therapy. Erlotinib is the recommended third line therapy for patients who have not received prior erlotinib or gefitinib. Data are insufficient to recommend the routine third line use of cytotoxic drugs. ${ }^{10}$

\section{Pharmacodynamics of pemetrexed}

The chemical name of pemetrexed is N-[4-[2-(2-amino-4,7dihydro-4-oxo-1H- pyrrolo [2,3-d] pyrimidin-5-yl) ethyl] benzoyl]-1-glutamic acid disodium salt, and the chemical structure of the drug is shown in Figure 1. Pemetrexed is a folate analog belonging to the antimetabolite class. The drug interferes with the synthesis of nucleic acids, resulting in a cytotoxic effect on neoplastic cells. Pemetrexed competes

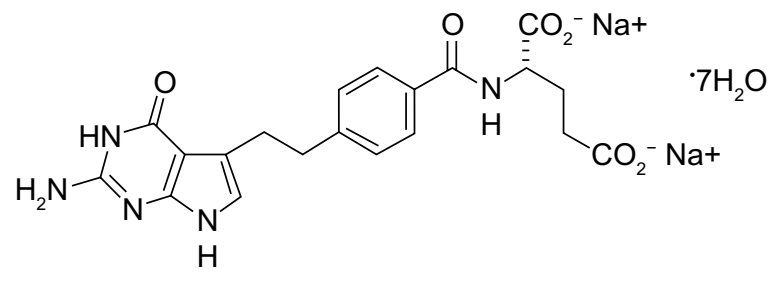

Figure I The structure of pemetrexed.

with reduced folate, thereby significantly inhibiting the activity of multiple folate requiring enzymes: thymidylate synthase (TS), dihydrofolate reductase (DHFR), and glycinamide ribonucleotide formyl transferase (GARFT). ${ }^{11,12}$ By inhibiting the formation of precursor purine and pyrimidine nucleotides, pemetrexed prevents the formation of DNA and RNA, which are required for the growth and survival of both normal cells and cancer cells. Pemetrexed is transported into cells by the reduced folate carrier and the membrane folate binding protein transport systems. Once in the cell, pemetrexed is rapidly and efficiently converted to polyglutamate forms by the enzyme folylpolyglutamate synthetase. The polyglutamate forms are retained in cells and are even more potent inhibitors of TS and GARFT. Polyglutamation is a time- and concentration-dependent process that occurs in tumor cells and, to a lesser extent, in normal tissues. Polyglutamated metabolites have an increased intracellular half-life, resulting in prolonged drug action in malignant cells. ${ }^{13}$

Otake et $\mathrm{al}^{14}$ were the first to show TS overexpression in NSCLC, in which $60.9 \%$ (14/23) of resected NSCLC samples displayed TS protein expression. ${ }^{14}$ Furthermore, they also performed the fluorodeoxyuridine- $5^{\prime}$-monophosphate binding assay and found that TS enzymatic activity ranged from 1.8 to $56.9 \mathrm{pmol} / \mathrm{g}$ protein in NSCLC samples, indicating that TS may be involved in NSCLC tumorigenesis. ${ }^{14}$ Nakagawa et al also found that NSCLC cell proliferation was associated with increased levels of TS expression, especially in lung adenocarcinoma cells. ${ }^{15}$ Wang et al's study further showed that the expression of TS mRNA and protein level in NSCLC tissues is higher than that in normal tissues ${ }^{16}$ which confirmed the findings of Otake et al ${ }^{14}$ and Nakagawa et al. ${ }^{15}$ The level of TS expression in squamous cell lung carcinoma was associated with poor tumor differentiation, which is consistent with a study reported by Ceppi et al. ${ }^{17}$

\section{Pharmacokinetics of pemetrexed}

Pemetrexed is administered by the intravenous route only, is rapidly distributed in the body, and reaches peak plasmatic levels within 30 minutes. Eighty-one percent of pemetrexed is bound to plasma proteins and is rapidly eliminated 
(half-life: 3.5 hours; total systemic clearance: $91.8 \mathrm{~mL} / \mathrm{min}$ ), primarily through the kidneys by glomerular filtration and active tubular secretion. Approximately $90 \%$ of pemetrexed is excreted in the urine within 24 hours after administration and only a limited amount of the drug is metabolized by the liver. Preclinical data suggest that pemetrexed does not interfere significantly with the metabolism of other drugs by cytochrome P450 isozymes. ${ }^{18}$

\section{Toxicity of pemetrexed}

Pemetrexed's toxicity is relatively mild. Scagliotti et al's Phase III study ${ }^{19}$ shows that the key hematologic grade 3 or 4 drug-related toxicities for cisplatin/pemetrexed were significantly lower than those in cisplatin/gemcitabine (neutropenia: $15 \%$ versus $27 \%$, anemia: $6 \%$ versus $10 \%$, and thrombocytopenia: $4 \%$ versus $13 \%$, respectively; $P<0.001) .{ }^{19}$ For cisplatin/pemetrexed versus cisplatin/gemcitabine, drug-related grade 3 or 4 febrile neutropenia (1\% versus $4 \%$, respectively; $P<0.002$ ) and alopecia (all grades, $12 \%$ versus $21 \%$, respectively; $P<0.001$ ) were also significantly lower. ${ }^{19}$ The primary adverse effect of pemetrexed, myelosuppression, presents as neutropenia and thrombocytopenia, is the dose-limiting toxicity of the drug, and is associated with elevated pretreatment levels of plasma homocysteine (a marker of folate deficiency). Plasma homocysteine predicts severe myelosuppression while a high pretreatment level of methylmalonic acid (a marker of vitamin B12 deficiency) is an independent predictor of severe diarrhea and mucositis. It has been reported that supplementation with folic acid and vitamin B12 substantially reduces pemetrexed-related toxicity without reducing efficacy. ${ }^{20}$ According to these findings, ingestion of folic acid (400 $\mu \mathrm{g} /$ day) and vitamin B12 $(1,000 \mu \mathrm{g}$ per three cycles) is very important for patients receiving pemetrexed. According to current recommendations, vitamin administration should start at least 1 week before the first cycle of pemetrexed and continue for at least 3 weeks after the last cycle of treatment. ${ }^{21}$ Erythema multiforme has been observed occasionally, but it may be prevented through prophylactic use of steroids; the suggested dose, 4 $\mathrm{mg}$ of dexamethasone twice daily, can be administered for 3 days, starting the day before infusion of pemetrexed. ${ }^{22}$

Nausea and vomiting have been described, however pemetrexed is classified as an agent with low emesis risk. ${ }^{23}$ In first line therapy pemetrexed is always administered in combination with other more emetogenic agents, such as cisplatin; therefore, adequate antiemetic prophylaxis is necessary. Because of the toxicity of some chemotherapeutic agents, a widely held misperception contends that all elderly patients, even those with good performance status (performance status [PS] 0-1), are unable to tolerate aggressive chemotherapy. Gridelli et al analyzed two randomized studies to evaluate the survival and safety of treatment with pemetrexed in elderly patients ( $<65$ years and $\geq 65$ years, and $<70$ years and $\geq 70$ years; all patients PS $0-1$ ) with nonsquamous NSCLC. ${ }^{24}$ They found that for patients with first line therapy, the incidence of grade $3 / 4$ toxicities related to pemetrexed plus cisplatin therapy relative to comparator-related toxicities in each of the four age groups were generally consistent with the results reported for all patients with nonsquamous NSCLC (neutropenia $15.1 \%$, thrombocytopenia $4.1 \%$, anemia $5.6 \%$, fatigue $6.7 \%$, and vomiting $6.1 \%$ ). The rates of most hematologic toxicities appeared to increase with age. The rates of neutropenia ranged from $11.5 \%$ in patients $<65$ years to $22.6 \%$ in the $\geq 70$ year age group. Thrombocytopenia rates ranged from $2.8 \%$ in patients $<65$ years to $7.2 \%$ in patients $\geq 70$ years. Febrile neutropenia rates were also higher in the older age groups $(0.5 \%$ in patients $<65$ years versus $2.8 \%$ in patients $\geq 65$ years). Anemia did not show an increase in rate with age and ranged from $2.4 \%$ in the oldest age group ( $\geq 70$ years) to $5.9 \%$ in the youngest age group ( $<65$ years). Fatigue rates were consistent throughout all age groups and ranged from $6.0 \%$ in patients $<70$ years to $10.7 \%$ in patients $\geq 70$ years. Nausea and vomiting also occurred at similar rates across all age groups, although the highest rate of vomiting (6.9\%) was observed in the youngest age group ( $<65$ years). The death rate due to therapy-related toxicity for this study was low. During the study and within 30 days of the last study drug dose, the toxic death rates according to age $(<65$ years and $\geq 65$ years and $<70$ years and $\geq 70$ years) were $0.9 \%$, $1.5 \%, 0.9 \%$, and $2.0 \%$, respectively. In patients with maintenance therapy the analysis results were similar. Therefore, the toxicities of pemetrexed in elderly patients were manageable, reversible, and consistent with the favorable safety profile. There was minimal variation in the hematologic toxicities between the age groups, and the majority of hematologic toxicity rates were low.

The elevation of transaminases and bilirubin has been reported in $10 \%-15 \%$ of treated patients; however, this elevation is usually transitory and asymptomatic. ${ }^{22}$ Because pemetrexed is mostly eliminated through the kidneys, creatinine clearance should be evaluated before infusion of pemetrexed; patients with creatinine clearance $>45 \mathrm{~mL} / \mathrm{min}$ do not need dose adjustment but pemetrexed should be avoided in patients with creatinine clearance $<45 \mathrm{~mL} / \mathrm{min}$, because the dose adjustment scheme is undecided. In addition, nonsteroidal anti-inflammatory drugs and aspirin may reduce the 
renal excretion of pemetrexed, potentially causing increased toxicity; therefore, administration of such drugs should be interrupted at least 2 days before pemetrexed and should not be restarted until at least 2 days after its administration. ${ }^{25}$

Methotrexate, which has a structure and pharmacokinetics similar to pemetrexed, is associated with increased toxicity in patients with serous cavity fluid (such as pleural effusions or ascites); however, pemetrexed is well tolerated in such patients and dose adjustment is not needed. ${ }^{26}$

\section{Clinical efficacy of pemetrexed Pemetrexed in first line therapy}

NSCLC is the most common cause of cancer mortality worldwide and accounts for approximately $85 \%$ of all lung cancer cases. ${ }^{27-29}$ Unfortunately, the great majority of patients are diagnosed with stage III or IV disease, and those with stage IV disease have a very dismal prognosis. Chemotherapeutic agents in the treatment of advanced NSCLC, including squamous cell carcinoma, adenocarcinoma, and large cell carcinoma, have reached a plateau of effectiveness when administered in the classic modality (4-6 cycles of treatment). Double agent platinum-based chemotherapy, which markedly prolongs MST, is the standard therapy for patients with advanced stage NSCLC and a preserved functional status, ${ }^{4,30}$ although nonplatinum-containing combinations are acceptable alternatives for patients who are not fit enough to receive platinum agents. ${ }^{31}$

Recently, a randomized Phase III trial comparing gemcitabine with pemetrexed as first line therapy for advanced NSCLC demonstrated that pemetrexed treatment provided health-related quality of life (HRQoL) and survival efficacy similar to gemcitabine with less hematologic toxicity and more convenience. ${ }^{32}$ A finding of nonsquamous histology significantly predicts the clinical effect of pemetrexed treatment, based on both prospective and retrospective analyses from separate trials. ${ }^{33-35}$

In 2008, Scagliotti et al $^{19}$ first compared the efficacy of first line treatment with cisplatin/pemetrexed versus cisplatin/gemcitabine in NSCLC patients. ${ }^{19}$ In this study, OS was identical for both arms (10.3 months) but survival was significantly longer with cisplatin/pemetrexed in the nonsquamous subgroups (12.6 versus 10.9 months in adenocarcinoma, 10.4 versus 6.7 months in large cell histology, respectively). ${ }^{19}$

Carboplatin can be used in patients who are unable to tolerate cisplatin. ${ }^{23}$ Zinner et al reported that the carboplatin/ pemetrexed combination is encouraging. ${ }^{36}$ A Phase III trial performed by The Norwegian Lung Cancer Study Group ${ }^{32}$ that enrolled 436 patients compared carboplatin/pemetrexed and carboplatin/gemcitabine as first line treatments for advanced NSCLC. ${ }^{32}$ The primary endpoint was HRQoL and the secondary endpoints were OS and toxicity. The two regimens achieved similar results in terms of HRQoL and OS (7.3 months for carboplatin/pemetrexed versus 7.0 months for carboplatin/gemcitabine; $P=0.63$ ). However, in the carboplatin/gemcitabine arm they found more grade 3 to 4 hematologic toxicity than in the carboplatin/pemetrexed arm, including leucopenia (46\% versus $23 \%$, respectively; $P<0.001)$, neutropenia ( $51 \%$ versus $40 \%$, respectively; $P=0.024)$, and thrombocytopenia (56\% versus $24 \%$, respectively; $P<0.001)$. In another randomized Phase III trial, the carboplatin/pemetrexed combination was compared with carboplatin/docetaxel in patients with advanced nonsquamous NSCLC. ${ }^{37}$ The primary endpoint was survival without toxicity, defined as the interval from randomization to the first treatment-induced grade 3-4 adverse event. Patients in the carboplatin/pemetrexed group had a longer median survival without toxicity than did patients in the carboplatin/docetaxel group (3.2 versus 0.7 months; hazard ratio [HR] $=0.45 ; 95 \%$ confidence interval [CI] 0.34-0.61). The median OS was similar (14.9 months with carboplatin/pemetrexed versus 14.7 months with carboplatin/docetaxel; $\mathrm{HR}=0.93 ; 95 \% \mathrm{CI}$ 0.66-1.32). In summary, these data suggest that carboplatin/ pemetrexed can provide an adequate first line regimen for nonsquamous NSCLC.

Based on these results, pemetrexed has been recommended as a first line treatment for patients with advanced nonsquamous NSCLC. So far, pemetrexed has shown activity and acceptable toxicity comparable to other third generation regimens in Caucasian patients with advanced nonsquamous NSCLC. ${ }^{38}$

\section{Pemetrexed in maintenance therapy}

Pemetrexed can also be used in maintenance therapy. A Phase III trial that compared pemetrexed with placebo as maintenance therapy after four cycles of platinum-based doublet chemotherapy (docetaxel, gemcitabine, or paclitaxel) found longer PFS (4.3 months with pemetrexed versus 2.6 months with placebo; HR $=0.50 ; 95 \%$ CI $0.42-0.61$ ) and OS (13.4 months with pemetrexed versus 10.6 months with placebo; HR $=0.79 ; 95 \%$ CI $0.65-0.95) .{ }^{39}$

In addition, the AVAPERL trial compared maintenance therapy with pemetrexed/bevacizumab to single agent bevacizumab after first line treatment with cisplatin, pemetrexed, and bevacizumab. The 253 patients who had stable disease or response to first line chemotherapy were chosen to receive bevacizumab or pemetrexed/bevacizumab. The 
pemetrexed/bevacizumab group had longer PFS (10.2 months for pemetrexed/bevacizumab versus 6.6 months for bevacizumab; HR $=0.50) .{ }^{40}$

The PARAMOUNT study compared pemetrexed maintenance therapy with placebo in patients with stage IV NSCLC treated with first line doublet chemotherapy using cisplatin and pemetrexed. It found a longer PFS with pemetrexed than with placebo (3.9 months with pemetrexed versus 2.6 months with placebo; $\mathrm{HR}=0.64 ; 95 \% \mathrm{CI}$ $0.51-0.81$ ). The grade $3-5$ toxicities of the two groups were similar, except for hematologic toxicities. The quality of life in the two groups was also similar. ${ }^{41}$

Furthermore, the ECOG 5508 trial $^{42}$ will compare three maintenance regimens (pemetrexed alone, bevacizumab alone, and the combination of pemetrexed and bevacizumab) following carboplatin, paclitaxel, and bevacizumab first line treatment; we are expecting the results.

\section{Pemetrexed in second line therapy}

In advanced NSCLC, most patients should be offered second line systemic therapy upon disease progression following first line therapy. The recommended drugs for second line treatment are docetaxel, pemetrexed, and epidermal growth factor receptor tyrosine kinase inhibitors. Single docetaxel is the established therapy for second line treatment of NSCLC. Pemetrexed for second line treatment of advanced NSCLC was validated through a Phase III randomized clinical trial, which compared pemetrexed with docetaxel. In this trial, Hanna et al proved the noninferiority of pemetrexed in comparison with docetaxel in second line treatment of NSCLC; median PFS was 2.9 months in both arms, and median survival time was 8.3 versus 7.9 months ( $P$-value was nonsignificant). ${ }^{43}$ A subset analysis of this study, conducted in elderly patients, showed similar results. ${ }^{44}$ In these two studies, docetaxel was chosen as a comparator because at that time, it was the only chemotherapeutic agent approved for second line treatment of NSCLC. By enrolling 571 patients throughout the world the primary endpoint (noninferiority of pemetrexed) was met. Similarly, median PFS was equal in both arms. Hematologic toxicity in patients treated with docetaxel was greater than those treated with pemetrexed. Specifically, patients who received docetaxel had a higher frequency of grade 3-4 neutropenia $(40.2 \%$ versus $5.3 \% ; P<0.001)$, including febrile neutropenia $(12.7 \%$ versus $1.9 \% ; P<0.001)$. Therefore, treatment with granulocyte colony-stimulating factor and hospitalization due to neutropenia were more frequent in the docetaxel arm. The incidence of thrombocytopenia was similar in the two arms ( $P$-value was not significant). Alopecia was less in patients with pemetrexed than in the docetaxel $\operatorname{arm}(6.4 \%$ versus $37.7 \% ; P<0.001)$, but the frequencies of other toxicities were similar.

A retrospective risk benefit analysis by Peterson et al confirmed that the safety profile of pemetrexed was more favorable than that of docetaxel and suggested that histological type modified the treatment effect. ${ }^{45}$ In squamous cell NSCLC, median survival was 6.2 months with pemetrexed and 7.4 months with docetaxel (HR $=1.563$; 95\% CI 1.079-2.264) and in nonsquamous NSCLC, median survival was 9.3 versus 8 months, respectively $(\mathrm{HR}=0.778$; 95\% CI 0.607-0.997). Based on these results, pemetrexed was approved for previously treated nonsquamous NSCLC patients. A Phase III study compared standard $\left(500 \mathrm{mg} / \mathrm{m}^{2}\right)$ versus high dose $\left(900 \mathrm{mg} / \mathrm{m}^{2}\right)$ pemetrexed as second line chemotherapy. ${ }^{46}$ There was no difference in median survival (6.7 versus 6.9 months; HR $=1.01$; 95\% CI 0.837-1.226) or in PFS (2.8 versus 2.6 months; HR $=0.97$; 95\% CI $0.817-1.147)$. A Phase II randomized trial previously proved the noninferiority of received pemetrexed, either $500 \mathrm{mg} /$ $\mathrm{m}^{2}$ (P500) or $1,000 \mathrm{mg} / \mathrm{m}^{2}$ (P1000). ${ }^{47} \mathrm{P} 1000$ and P500 did not differ significantly in terms of response rate $(18.5 \%$ for P1000 versus $14.8 \%$ for P500; $P=0.58)$. P500 was similar to P1000 in terms of median PFS (3.0 versus 2.5 months; $P=0.71$ ) and median OS (16 versus 12.6 months; $P=0.14$ ). Both schedules were well tolerated, although P500 generally produced milder toxicity. Currently, available data support the use of pemetrexed at the dose of $500 \mathrm{mg} / \mathrm{m}^{2}$ as second line treatment.

In additional, pemetrexed has been used for malignant pleural mesothelioma and advanced peritoneal mesothelioma, as reviewed by Boons and Nakano. ${ }^{48,49}$ There is inadequate proof that pemetrexed can be used for small cell lung cancer, and the efficacy and safety of these therapies are yet to be established.

\section{Cost effectiveness of pemetrexed}

Pemetrexed is a very costly drug. Since nonsquamous NSCLC patients may administer this drug for first line therapy, maintenance therapy, and second line therapy, it is administered for a long period of time. Therefore, the treatment cost of pemetrexed is a high priority issue. A Phase III trial ${ }^{50}$ was conducted to compare the cost effectiveness of pemetrexed and docetaxel in second line therapy of NSCLC. Docetaxel was proved to be associated with a lower treatment period cost $(€ 9,709 \pm € 6,272$ for docetaxel versus $€ 13,436 \pm € 6,508$ for pemetrexed; $P<0.001) .{ }^{50}$ 
Furthermore, Jakel et al conducted a systematic review to compare the cost effectiveness of pemetrexed, docetaxel, and erlotinib with best supportive care in second line or later line treatment of NSCLC. In the final results, erlotinib and pemetrexed were both premeditated to be cost effective compared to docetaxel by the National Institute for Health and Clinical Excellence and Scottish Medicines Consortium. Erlotinib was also further considered to be cost effective compared to the best supportive care by the Pharmaceutical Benefits Advisory Committee. ${ }^{5}$

\section{Predictive biomarkers for pemetrexed based chemotherapy}

Despite great improvements in the treatment of NSCLC over the past several years, the prognosis for patients with advanced disease is still poor. Chemotherapy resistance is a key determinant of the dismal prognoses for lung cancer patients. It is known that lung cancer is driven by genomic alterations; cancer cells use multiple mechanisms to alter the activity of key genes including mutation, amplification, deletion, intrachromosomal and interchromosomal translocation, and epigenetic mechanisms. Advances in the field of genomics during the past decade have greatly increased our understanding of the genomic alterations that contribute to lung cancer, but additional challenges must be addressed before the goal of personalized cancer therapy can become a reality for lung cancer patients. The clinical relevance for pemetrexed metabolism of genetic polymorphisms of the folate pathway genes has not been fully elucidated. Several genetic variations associated with the folate metabolic pathway and downstream events have been correlated with clinical outcomes in patients treated with pemetrexed. ${ }^{51-53}$

The evolving newly recognized field of microRNA (miRNA) offers insight into an additional regulatory layer affecting drug response. miRNAs have been implicated in a wide array of fundamental biological processes, such as cell proliferation, differentiation, and apoptosis and thus may function as oncogenes or tumor suppressing genes. Accumulating evidence shows that miRNAs are grossly dysregulated in human cancers, including NSCLC. Importantly, circulating miRNAs have been detected as potential bloodbased biomarkers for cancer detection. The analysis of circulating miRNA profiles may improve not only the knowledge of miRNA-mediated mechanisms, but may also predict outcome for cancer patients. ${ }^{54}$

Recent works have found that DHFR, the target enzyme inhibited by pemetrexed, is regulated at the translational level by miR-24. ${ }^{51}$ Further findings revealed that miR-22 and
miR-34a target methylenetetrahydrofolate reductase, a key enzyme in folate metabolism. ${ }^{55}$ Methylenetetrahydrofolate reductase polymorphisms have been associated with clinical outcomes in NSCLC patients treated with pemetrexed. ${ }^{51-53}$ Therefore, miR-22, miR-24, and miR-34a may be potential biomarkers to predict pemetrexed response. Moreover, upregulation of miR-22, miR-24, and miR-34a in lung cancer patients could provide useful biomarkers to follow early stage NSCLC and innovative approaches to early diagnosis in healthy heavy smokers. MicroRNAs are stable in the sputum of cancer patients; therefore, they are promising candidates for biomarkers. ${ }^{56}$

As we mentioned before, pemetrexed can significantly inhibit the activity of multiple folate-requiring enzymes, including TS. Wang's study found an association between TS gene 3'-UTR 1494del 6 bp polymorphisms and outcome of pemetrexed treatment in lung adenocarcinoma patients; the PFS and OS of patients with ( $-6 \mathrm{bp} /-6 \mathrm{bp}$ ) and $(-6 \mathrm{bp} /+6 \mathrm{bp})$ genotypes were significantly different. The multivariate analysis showed that older age, advanced disease stage, and the TS $3^{\prime}$-UTR 1494bp ( -6 bp/+6 bp) genotype were prognostic for a poorer outcome. They concluded that "TS is highly expressed in NSCLC and that polymorphisms of TS $3^{\prime}$-UTR 1494del $6 \mathrm{bp}$ are associated with lung adenocarcinoma patients' sensitivity to pemetrexed treatment." ${ }^{\prime 16}$ In their conclusion they suggest that "TS gene polymorphisms should be further evaluated as prognostic markers for personalized chemotherapy in lung adenocarcinoma". ${ }^{16}$

In the past decades, major improvements have been achieved in the treatment of advanced NSCLC. The current standard of first line therapy for advanced NSCLC consists of platinum-based doublets for a maximum of six cycles in the absence of unacceptable toxicity or progressive disease. ${ }^{7}$ Although $70 \%-80 \%$ of patients who receive first line chemotherapy experience the clinical benefits of response or stable disease, ${ }^{57-59}$ up to $50 \%$ of patients will eventually receive second line therapy and the 5 -year survival rate is less than $5 \% .{ }^{60}$ These treatment outcomes indicate an obvious need to improve the current treatment paradigm.

\section{Conclusion}

Pemetrexed is one of the most frequently used drugs in the treatment of nonsquamous NSCLC patients. It works by inhibiting DHFR, GARFT, and TS, thereby halting the synthesis of tumor cell DNA and RNA. Adenocarcinomas are more sensitive to pemetrexed; squamous cell carcinomas of the lung are less sensitive to the action of pemetrexed. 
This drug's good efficacy and the low risk profile make it one of the most commonly used first line treatments in combination with cisplatin or carboplatin for patients with nonsquamous NSCLC. This combination was more effective than a standard schedule (cisplatin/gemcitabine) in terms of PFS and OS. Pemetrexed is also widely used for maintenance therapy, produces improved PFS and OS, and is a suitable option after first line treatment for selected patients. The mechanism of action of pemetrexed is well known, which explains the efficacy of pemetrexed for nonsquamous NSCLC. Pemetrexed is also used as a single agent for second line therapy; it is a valid alternative for patients who did not receive the drug for first line chemotherapy. TS as a factor predictive of pemetrexed efficacy is yet to be demonstrated, although further studies are being conducted. The role of pemetrexed in the management of nonsquamous NSCLC may extend to the adjuvant setting and also to combination with radiotherapy in locally advanced disease; trials evaluating these roles are ongoing.

\section{Funding}

This research was supported partially by grants from the National Natural Science Foundation of China (81172233), the Science and Technology Support Key Program of Tianjin (12ZCDZSY16100), the Tianjin Natural Science Foundation (12JCZDJC24400), and the project of Ministry of Education for New Century Excellent Talents (NCET10-0956).

\section{Author contributions}

$\mathrm{XL}$ and JC wrote the paper. SW contributed materials and participated to revise the draft for important content.

\section{Disclosure}

The authors declare that no competing interests exist.

\section{References}

1. Jemal A, Murray T, Samuels A, Ghafoor A, Ward E, Thun MJ. Cancer statistics, 2003. CA Cancer J Clin. 2003;53(1):5-26.

2. Smith W, Khuri FR. The care of the lung cancer patient in the 21st century: a new age. Semin Oncol. 2004;31(2 Suppl 4):11-15.

3. Chemotherapy in non-small cell lung cancer: a meta-analysis using updated data on individual patients from 52 randomised clinical trials. Non-small Cell Lung Cancer Collaborative Group. BMJ. 1995; 311(7010):899-909.

4. Schiller JH, Harrington D, Belani CP, et al. Comparison of four chemotherapy regimens for advanced non-small-cell lung cancer. N Engl J Med. 2002;346(2):92-98.

5. Jakel A, Plested M, Dharamshi K, Modha R, Bridge S, Johns A. A systematic review of economic evaluations in second and later lines of therapy for the treatment of non-small cell lung cancer. Appl Health Econ Health Policy. 2013;11(1):27-43.
6. Tomasini P, Greillier L, Khobta N, Barlesi F. The place of pemetrexed in the management of non-small-cell lung cancer patients. Expert Rev Anticancer Ther. 2013;13(3):257-266.

7. Azzoli CG, Baker S Jr, Temin S, et al; American Society of Clincal Oncology. American Society of Clinical Oncology Clinical Practice Guideline update on chemotherapy for stage IV non-small-cell lung cancer. J Clin Oncol. 2009;27(36):6251-6266.

8. Azzoli CG, Temin S, Aliff T, et al; American Society of Clincal Oncology. 2011 Focused Update of 2009 American Society of Clinical Oncology Clinical Practice Guideline Update on Chemotherapy for Stage IV Non-Small-Cell Lung Cancer. J Clin Oncol. 2011;29(28): 3825-3831.

9. Azzoli CG, Temin S, Giaccone G. 2011 Focused Update of 2009 American Society of Clinical Oncology Clinical Practice Guideline Update on Chemotherapy for Stage IV Non-Small-Cell Lung Cancer. J Oncol Pract. 2012;8(1):63-66.

10. Quan L, Chen W, Shu Y. [Current status and prospects of maintenance therapy in advanced stage non-small cell lung cancer]. Zhongguo Fei Ai Za Zhi. 2010;13(6):637-641. Chinese.

11. Chattopadhyay S, Moran RG, Goldman ID. Pemetrexed: biochemical and cellular pharmacology, mechanisms, and clinical applications. Mol Cancer Ther. 2007;6(2):404-417.

12. Racanelli AC, Rothbart SB, Heyer CL, Moran RG. Therapeutics by cytotoxic metabolite accumulation: pemetrexed causes ZMP accumulation, AMPK activation, and mammalian target of rapamycin inhibition. Cancer Res. 2009;69(13):5467-5474.

13. Claudine M. Baldwin, Caroline M. Perry. Pemetrexed. Drugs. 2009; 69:(16) 2279-2302.

14. Otake Y, Tanaka F, Yanagihara K, et al. Expression of thymidylate synthase in human non-small cell lung cancer. Jpn J Cancer Res. 1999;90(11):1248-1253.

15. Nakagawa T, Otake Y, Yanagihara K, et al. Expression of thymidylate synthase is correlated with proliferative activity in non-small cell lung cancer (NSCLC). Lung Cancer. 2004;43(2):145-149.

16. Wang X, Wang Y, Wang Y, Cheng J, Wang Y, Ha M. Association of thymidylate synthase gene $3^{\prime}$-untranslated region polymorphism with sensitivity of non-small cell lung cancer to pemetrexed treatment: TS gene polymorphism and pemetrexed sensitivity in NSCLC. J Biomed Sci. 2013;20:5.

17. Ceppi P, Volante M, Saviozzi S, et al. Squamous cell carcinoma of the lung compared with other histotypes shows higher messenger RNA and protein levels for thymidylate synthase. Cancer. 2006;107(7): 1589-1596.

18. Sorensen JB. Pharmacokinetic evaluation of pemetrexed. Expert Opin Drug Metab Toxicol. 2011;7(7):919-928.

19. Scagliotti GV, Parikh P, von Pawel J, et al. Phase III study comparing cisplatin plus gemcitabine with cisplatin plus pemetrexed in chemotherapy-naive patients with advanced-stage non-small-cell lung cancer. J Clin Oncol. 2008;26(21):3543-3551.

20. Niyikiza C, Baker SD, Seitz DE, et al. Homocysteine and methylmalonic acid: markers to predict and avoid toxicity from pemetrexed therapy. Mol Cancer Ther. 2002;1(7):545-552.

21. Kim YS, Sun JM, Ahn JS, Ahn MJ, Park K. The optimal duration of vitamin supplementation prior to the first dose of pemetrexed in patients with non-small-cell lung cancer. Lung Cancer. 2013;81(2): 231-235.

22. ALIMTA [package insert]. Indianopolis, IN; Eli Lilly and Company; 2012. Available from: http://www.accessdata.fda.gov/drugsatfda docs/label/2012/021462s0391bl.pdf. Accessed February 24, 2014.

23. NCCN Clinical practice guidelines in oncology: Antiemesis. Version 1. Journal of the National Comprehensive Cancer Network 2012;10(4):456-485.

24. Gridelli C, Brodowicz T, Langer CJ, et al. Pemetrexed therapy in elderly patients with good performance status: analysis of two phase III trials of patients with nonsquamous non-small-cell lung cancer. Clin Lung Cancer. 2012;13(5):340-346. 
25. Yang TY CG, Chen KC, et al. Pemetrexed induces both intrinsic and extrinsic apoptosis through ataxia telangiectasia mutated/p53-dependent and -independent signaling pathways. Mol Carcinog. 2013;52(3): 183-194.

26. Dickgreber NJ, Sorensen JB, Paz-Ares LG, et al. Pemetrexed safety and pharmacokinetics in patients with third-space fluid. Clin Cancer Res. 2010;16(10):2872-2880.

27. Shepherd FA. Screening, diagnosis, and staging of lung cancer. Curr Opin Oncol. 1993;5(2):310-322.

28. Walling J. Chemotherapy for advanced non-small-cell lung cancer. Respir Med. 1994;88(9):649-657.

29. Feigenberg SJ, Hanlon AL, Langer C, et al. A phase II study of concurrent carboplatin and paclitaxel and thoracic radiotherapy for completely resected stage II and IIIA non-small cell lung cancer. J Thorac Oncol. 2007;2(4):287-292.

30. Pfister DG, Johnson DH, Azzoli CG, et al; American Society of Clinical Oncology. American Society of Clinical Oncology treatment of unresectable non-small-cell lung cancer guideline: update 2003. $J$ Clin Oncol. 2004;22(2):330-353.

31. Breathnach OS, Freidlin B, Conley B, et al. Twenty-two years of phase III trials for patients with advanced non-small-cell lung cancer: sobering results. J Clin Oncol. 2001;19(6):1734-1742.

32. Gronberg BH, Bremnes RM, Flotten O, et al. Phase III study by the Norwegian lung cancer study group: pemetrexed plus carboplatin compared with gemcitabine plus carboplatin as first-line chemotherapy in advanced non-small-cell lung cancer. J Clin Oncol. 2009;27(19):3217-3224.

33. Scagliotti G, Hanna N, Fossella F, et al. The differential efficacy of pemetrexed according to NSCLC histology: a review of two Phase III studies. Oncologist. 2009;14(3):253-263.

34. Zinner RG, Novello S, Peng G, Herbst R, Obasaju C, Scagliotti G. Comparison of patient outcomes according to histology among pemetrexed-treated patients with stage IIIB/IV non-small-cell lung cancer in two phase II trials. Clin Lung Cancer. 2010;11(2):126-131.

35. $\mathrm{Hu} \mathrm{X}$, Jiao S, Zhang S, et al. [Efficacy and toxicity of pemetrexed or gemcitabine combined with cisplatin in the treatment of patients with advanced non-small cell lung cancer]. Zhongguo Fei Ai Za Zhi. 2012;15(10):569-575. Chinese.

36. Zinner RG, Fossella FV, Gladish GW, et al. Phase II study of pemetrexed in combination with carboplatin in the first-line treatment of advanced nonsmall cell lung cancer. Cancer. 2005;104(11):2449-2456.

37. Rodrigues-Pereira J, Kim JH, Magallanes M, et al. A randomized phase 3 trial comparing pemetrexed/carboplatin and docetaxel/carboplatin as first-line treatment for advanced, nonsquamous non-small cell lung cancer. J Thorac Oncol. 2011;6(11):1907-1914.

38. Manegold C, Gatzemeier U, von Pawel J, et al. Front-line treatment of advanced non-small-cell lung cancer with MTA (LY231514, pemetrexed disodium, ALIMTA) and cisplatin: a multicenter phase II trial. Ann Oncol. 2000;11(4):435-440.

39. Ciuleanu T, Brodowicz T, Zielinski C, et al. Maintenance pemetrexed plus best supportive care versus placebo plus best supportive care for non-small-cell lung cancer: a randomised, double-blind, phase 3 study. Lancet. 2009;374(9699):1432-1440.

40. Barlesi F, Scherpereel A, Rittmeyer A, et al. Randomized phase III trial of maintenance bevacizumab with or without pemetrexed after first-line induction with bevacizumab, cisplatin, and pemetrexed in advanced nonsquamous non-small-cell lung cancer: AVAPERL (MO22089). J Clin Oncol. 2013;31(24):3004-3011.

41. Paz-Ares LG, Gomez-Roca C, Delord JP, et al. Phase I pharmacokinetic and pharmacodynamic dose-escalation study of RG7160 (GA201), the first glycoengineered monoclonal antibody against the epidermal growth factor receptor, in patients with advanced solid tumors. J Clin Oncol. 2011;29(28):3783-3790.

42. ClinicalTrials.gov. Eastern Cooperative Oncology Group. Bevacizumab or Pemetrexed Disodium Alone or In Combination After Induction Therapy in Treating Patients With Advanced Non-Squamous NonSmall Cell Lung Cancer. Available from: http://www.clinicaltrials.gov/ ct $2 /$ show/NCT01107626?term=ECOG+5508+TRIAL\&rank=1. NLM identifier: NCT01107626. Accessed March 24, 2014.
43. Hanna N, Shepherd FA, Fossella FV, et al. Randomized phase III trial of pemetrexed versus docetaxel in patients with non-small-cell lung cancer previously treated with chemotherapy. J Clin Oncol. 2004;22(9): 1589-1597.

44. Weiss GJ, Langer C, Rosell R, et al. Elderly patients benefit from second-line cytotoxic chemotherapy: a subset analysis of a randomized phase III trial of pemetrexed compared with docetaxel in patients with previously treated advanced non-small-cell lung cancer. J Clin Oncol. 2006;24(27):4405-4411.

45. Peterson P PK, Fossella F, Gatzemeier U, John W, Scagliotti G. Is pemetrexed more effective in adenocarcinoma and large cell lung cancer than in squamous cell carcinoma? A retrospective analysis of a Phase III trial of pemetrexed versus docetaxel in previously treated non-small cell lung cancer. J Thorac Oncol. 2007; 2(supp14): S851.

46. Cullen MH, Zatloukal P, Sorenson S, et al. A randomized phase III trial comparing standard and high-dose pemetrexed as second-line treatment in patients with locally advanced or metastatic non-small-cell lung cancer. Ann Oncol. 2008;19(5):939-945.

47. Ohe Y, Ichinose Y, Nakagawa K, et al. Efficacy and safety of two doses of pemetrexed supplemented with folic acid and vitamin B12 in previously treated patients with non-small cell lung cancer. Clin Cancer Res. 2008;14(13):4206-4212.

48. Boons CC, VAN tulder MW, Burgers JA, Beckeringh JJ, Wagner C, Hugtenburg JG. The value of pemetrexed for the treatment of malignant pleural mesothelioma: a comprehensive review. Anticancer Res. 2013;33(9):3553-3561.

49. Nakano M, Kusaba H, Makiyama A, et al. Pemetrexed combined with platinum-based chemotherapy for advanced malignant peritoneal mesothelioma: retrospective analysis of six cases. Anticancer Res. 2014;34(1):215-220.

50. Vergnenegre A, Corre R, Berard H, et al; 0506 GFPC Team. Cost-effectiveness of second-line chemotherapy for non-small cell lung cancer: an economic, randomized, prospective, multicenter phase III trial comparing docetaxel and pemetrexed: the GFPC 05-06 study. J Thorac Oncol. 2011;6(1):161-168.

51. Hou J, Lambers M, den Hamer B, et al. Expression profiling-based subtyping identifies novel non-small cell lung cancer subgroups and implicates putative resistance to pemetrexed therapy. J Thorac Oncol. 2012;7(1):105-114.

52. Franchina T, Proto C, Chiofalo G, et al. Folate pathway implications in advanced Non Small Cell Lung Cancer (NSCLC): Impact of thymidylate synthase (TS) promoter and methlylenetetrahydrofolate reductase (MTHFR) C677T and A1298C variants expression on patients' outcome and correlation with p53 codon 72 mutations. In: Programs and abstracts of the 14th World Conference on Lung Cancer. Amsterdam; The Netherlands.

53. Kim JYJ, Kong J, Lee S, et al. Correlation of genetic polymorphisms in folate metabolic pathway genes with clinical outcomes in pemetrexedtreated advanced NSCLC patients. J Clin Oncol. 2010;28:15.

54. Gao W, Liu L, Lu X, Shu Y. Circulating microRNAs: possible prediction biomarkers for personalized therapy of non-small-cell lung carcinoma. Clin Lung Cancer. 2011;12(1):14-17.

55. Shookhoff JM, Gallicano GI. A new perspective on neural tube defects: folic acid and microRNA misexpression. Genesis. 2010;48(5): 282-294.

56. D’Urso V, Doneddu V, Marchesi I, et al. Sputum analysis: non-invasive early lung cancer detection. J Cell Physiol. 2013;228(5):945-951.

57. Leighl NB, Paz-Ares L, Douillard JY, et al. Randomized phase III study of matrix metalloproteinase inhibitor BMS-275291 in combination with paclitaxel and carboplatin in advanced non-small-cell lung cancer: National Cancer Institute of Canada-Clinical Trials Group Study BR.18. J Clin Oncol. 2005;23(12):2831-2839.

58. Williamson SK, Crowley JJ, Lara PN Jr, et al; Southwest Oncology Group Trial S0003. Phase III trial of paclitaxel plus carboplatin with or without tirapazamine in advanced non-small-cell lung cancer: Southwest Oncology Group Trial S0003. J Clin Oncol. 2005;23(36): 9097-9104 
59. Reck M, von Pawel J, Zatloukal P, et al. Phase III trial of cisplatin plus gemcitabine with either placebo or bevacizumab as first-line therapy for nonsquamous non-small-cell lung cancer: AVAil. J Clin Oncol. 2009;27(8):1227-1234.
60. Correale P, Tindara Miano S, Remondo C, et al. Second-line treatment of non small cell lung cancer by biweekly gemcitabine and docetaxel $+/-$ granulocyte-macrophage colony stimulating factor and low dose aldesleukine. Cancer Biol Ther. 2009;8(6):497-502.

\section{Publish your work in this journal}

OncoTargets and Therapy is an international, peer-reviewed, open access journal focusing on the pathological basis of all cancers, potential targets for therapy and treatment protocols employed to improve the management of cancer patients. The journal also focuses on the impact of management programs and new therapeutic agents and protocols on

\section{Dovepress}

patient perspectives such as quality of life, adherence and satisfaction. The manuscript management system is completely online and includes a very quick and fair peer-review system, which is all easy to use. Visit http://www.dovepress.com/testimonials.php to read real quotes from published authors.

Submit your manuscript here: http://www.dovepress.com/oncotargets-and-therapy-journal 